\title{
Tranexamic Acid in Neuroanesthesia and Neurocritical Care: Time for Its Critical Appraisal
}

\author{
Bhavna Hooda ${ }^{1}$ Radhakrishnan Muthuchellappan² \\ ${ }^{1}$ Department of Anaesthesiology and Critical Care, Army Hospital \\ Research \& Referral, New Delhi, India \\ ${ }^{2}$ Department of Neuroanaesthesia and Neurocritical Care, \\ National Institute of Mental Health and Neurosciences (NIMHANS), \\ Bengaluru, Karnataka, India
}

\begin{abstract}
Address for correspondence Bhavna Hooda, MD, DM, Department of Anaesthesiology and Critical Care, Army Hospital Research \& Referral, New Delhi 110010, India (e-mail: bhavnahooda2007@rediffmail.com).
\end{abstract}

J Neuroanaesthesiol Crit Care 2019;6:257-266

\author{
Abstract \\ Keywords \\ - tranexamic acid \\ - traumatic brain injury \\ - intracerebral \\ hemorrhage \\ - chronic subdural \\ hematoma \\ - subarachnoid \\ hemorrhage \\ - perioperative period
}

There is a renewed interest in the use of antifibrinolytics, especially tranexamic acid (TxA), in varied clinical settings such as trauma, perioperative bleeding, cardiac surgery, and multilevel spine instrumentation procedures. Because of its prothrombotic potential, the use of TxA in neurosurgical disorders has not gained popularity since this subset of the population is more prone to developing thromboembolic complications. However, recent studies have shown that TxA reduces blood loss, reduces the need for allogeneic blood transfusion, and is associated with minimal side effects. In this narrative review, Google Scholar, PubMed, and EMBASE databases were searched to identify publications relevant to the current use of TxA in varied neurosurgical and critical care settings. These publications were analyzed, and a summary of the findings and the role of future research on this drug are presented in this review.

\section{Introduction}

Tranexamic acid (TxA) is a synthetic lysine analogue that competitively blocks the lysine-binding residues on fibrin clot and prevents the conversion of plasminogen to plasmin, thus inhibiting clot lysis. ${ }^{1}$ The middle of the twentieth century was the era of controlling surgical bleed using chemical hemostatic agents, with a keen interest in antifibrinolytics (AF). There was overwhelming literature on AF use in various surgical specialties with the aim of reducing perioperative bleed and transfusion requirements, particularly in cardiac surgical patients. Aprotinin, epsilon-amino-caproic acid (EACA), and TxA were the commonly studied AF agents. The spirits were soon dampened as reports of an association between aprotinin and renal failure and increased mortality trickled in, and this drug was banned in 2007. ${ }^{2}$ The ubiquitous use of AF was viewed with doubts about the possible life-threatening adverse effects. Similarly, there was a topsy-turvy use of AFs in the 1970s and 1980s in neurosurgery with a barrage of trials on its use in conservative management of cerebral aneurysms to prevent rebleeding. ${ }^{3}$ Over the next decade, administration of TxA in aneurysmal subarachnoid

received

February 5, 2019

accepted after revision

April 22, 2019

published online

July 26, 2019 hemorrhage (SAH) was marred by controversy with reports of increased risk of delayed cerebral ischemia. ${ }^{4}$ The largest trauma trial of this century, CRASH-2 (Clinical Randomisation of an Antibrinolytic in Significant Haemorrhage 2) and the nested CRASH-2 IBS (Intracranial Bleeding Study) opened the floodgates and revived this old drug with renewed interest in the field of trauma including head trauma. ${ }^{5,6}$ There are over 400 publications of its use in neurosurgery, and the spectrum of its use is ever expanding in conditions such as chronic subdural hematoma (CSDH), spontaneous intracerebral hemorrhage (ICH), SAH, skull base and vascular tumors, craniosynostosis, and corrective spine surgery. In this narrative review, Google Scholar, PubMed, and EMBASE databases were searched to identify publications relevant to the current use of TxA in varied neurosurgical and critical care settings. The authors begin this review with a brief discussion on the physiology of fibrinolysis and pharmacology of TxA, followed by its use in various neurosurgical conditions. In the end, this review will highlight the complications associated with TxA administration and conclude the review by discussing ongoing and future research on TxA with suggestions for its use in neuroanesthesia and critical care practices. 


\section{Fibrinolytic System}

Coagulation and fibrinolysis are the two pillars of the hemostatic system that work in perfect harmony to maintain vascular integrity, clot localization, and vascular patency.

Tissue injury stimulates the coagulation cascade with thrombin burst, platelet activation, and adhesion and conversion of fibrinogen to fibrin that in turn polymerizes to form a stable fibrin clot. This fibrin clot provides a hemostatic seal at the site of wall breach.

Simultaneously, the fibrinolytic system gets activated to localize the clot. Plasminogen and tissue plasminogen activator ( $\mathrm{tPA}$ ) complex bind to the lysine residues on the fibrin clot and generate the active serine protease, plasmin. Plasmin lyses the fibrin clot to soluble fibrin degradation products and D-dimers. ${ }^{7}$ The fibrinolytic activity is kept in check by the circulating serine protease inhibitors that include plasminogen activator inhibitors 1,2 , and $3, \alpha_{2}$-antiplasmin, $\alpha_{2}$-macroglobulin, and thrombin-activated fibrinolysis inhibitor.

This fine-tuned hemostatic system goes haywire in the event of massive trauma, surgery, and cardiopulmonary bypass resulting in excessive systemic fibrinolysis and coagulopathy. ${ }^{8}$ This speculation forms the basis for AF use in the above-mentioned settings.

\section{Tranexamic Acid Pharmacology}

Tranexamic acid was independently described by Okomato et $\mathrm{al}^{9}$ and Melander et al. ${ }^{10}$ TxA is a trans-stereoisomer of a synthetic derivative of amino acid lysine (trans-4-aminomethyl-cyclohexane-carboxylic acid: AMCHA). ${ }^{11}$ Being 7 to 10 times more potent than its congener EACA, it was soon embraced into clinical practice for blood conservation. ${ }^{1}$ With a half-life of 80 to 100 minutes, one-third of the drug is eliminated unchanged in the urine within 1 hour and over $90 \%$ in 24 hours. It crosses the blood-brain barrier (BBB) with cerebrospinal fluid (CSF) concentration reaching up to one-tenth of the plasma concentration. ${ }^{1,2}$ Though a complete fibrinolytic shutdown occurs at a plasma concentration of 100 to 150 $\mathrm{mg} / \mathrm{L}$, it has been suggested that $80 \%$ fibrinolysis inhibition is sufficient for a clinically significant effect and is achieved at a plasma concentration of $10 \mathrm{mg} / \mathrm{L} .^{12,13}$ In clinical practice, wide range of dosages have been described for TxA use in various clinical settings. However, it has been suggested that a total dose of $14 \mathrm{mg} / \mathrm{kg}$ or $1 \mathrm{~g}$ of TxA is adequate for blocking fibrinolysis and use of higher doses may be unnecessary. ${ }^{14}$ A recent trial, based on in vivo pharmacokinetic/pharmacodynamic model for TxA, suggested lower TxA concentrations (up to $20 \mathrm{mg} / \mathrm{L}$ ) to inhibit tPA fibrinolysis cascade. However, higher concentrations (up to $150 \mathrm{mg} / \mathrm{L}$ ) are required to inhibit urokinase-type plasminogen activator (uPA)-induced fibrinolysis and proinflammatory potential of plasmin. ${ }^{15}$

\section{Trauma}

One-third of the mortality in trauma patients is due to hemorrhage. It is estimated that coagulopathic trauma patients are eight times more likely to die in the first 24 hours of injury. ${ }^{16}$

\section{Rationale}

The earlier notion was that overzealous resuscitation and hypothermia-coagulopathy-acidosis (HCA) triad was the primary driver of bleeding in trauma patients. However, current research has shifted the focus toward an endogenous process of acute trauma-induced coagulopathy (ATC) as the root cause of bleeding and mortality in trauma patients. ${ }^{17}$ ATC commences within minutes of injury as a thrombin burst and is accompanied by a massive endothelial release of tPA, which manifests as disseminated intravascular coagulation (DIC) with a fibrinolytic phenotype. Over hours, this process translates into a state of fibrinolytic shutdown as the plasminogen inhibitors take control of the systemic fibrinolysis. ${ }^{18}$ It is noted that up to $35 \%$ of the trauma patients are coagulopathic at admission to the emergency department. In a thromboelastometry (ROTEM)-based analysis of 300 trauma patients, it was noted that more than one-half of the patients had elevated plasmin-antiplasmin complex levels, suggesting fibrinolytic activation. These patients experienced higher 28 -day mortality ( $12 \%$ vs. $1 \%, p<0.001){ }^{19}$

\section{Evidence}

This insight into the pathogenesis of trauma, with a window of hyperfibrinolysis, was identified as a potential target for AF application. It paved the way for the largest civilian trauma trial, CRASH-2, that enrolled over 20,000 adult trauma patients within 8 hours of injury across 274 hospitals in 40 countries. ${ }^{5}$ Trauma patients with significant hemorrhage or at risk of hemorrhage were randomly allocated to receive either TxA $1 \mathrm{~g}$ loading dose, followed by another $1 \mathrm{~g}$ over the next 8 hours or placebo. The all-cause mortality at 28 days was significantly lower in the TxA group compared with the placebo ( $14.5 \%$ vs. $16 \%$ ). The authors observed maximal survival benefit in patients who received TxA within 3 hours of injury (mortality relative risk [RR] 0.68 [ $\leq 1$ hour of injury] and 0.79 [1-3 hours of injury] . ${ }^{20}$ It is likely that trauma patients beyond the 3-hour window might have been more acidotic, hypothermic, and in a prothrombotic or fibrinolytic shutdown phase of the injury. The CRASH-2 trial, however, has been marred by serious criticism for various reasons. These include randomization design based on the uncertainty principle, the study population including patients from low- to medium-income group countries with varying levels of trauma systems, an absolute reduction in mortality by $0.8 \%$ due to bleeding between groups with no reduction in transfusion requirements, lack of data on injury severity scores, laboratory tests for fibrinolysis, coagulation derangements or inadequate perfusion (acidosis, lactate), and $100 \%$ follow-up in spite of the vast spread of study population. MATTERs (Military Application of Tranexamic acid in Trauma Emergency Resuscitation) study, a retrospective observational study conducted in Afghanistan, enrolled combat trauma patients requiring blood transfusion. ${ }^{21}$ In a total of 293 patients who received TxA, the unadjusted mortality was lower in TxA group despite being more severely injured (TxA vs. non-TxA group; $17.4 \%$ vs. $23.9 \%$, respectively; $p=0.03$ ). This effect was more evident in the subgroup requiring massive blood transfusion ( $14.4 \%$ vs. $28.1 \%$, respectively; 
$p=0.004)$. TxA administration was associated with better survival (odds ratio $[\mathrm{OR}]=7.23$; 95\% confidence interval $[\mathrm{CI}]$ : $3.016-17.322)$ and less coagulopathy $(p=0.003)$. This study demonstrated a clear survival benefit with a number needed to treat (NNT) of 1:7 compared with the larger CRASH-2 trial where the NNT was approximately 1:67. A Cochrane review explored the effect of AFs in acute traumatic injury and concluded that AFs reduced the risk of death from any cause (RR $=0.90,95 \%$ CI: 0.85-0.96). ${ }^{22}$

The Antifibrinolytics Collaboration trial did a metaanalysis on 40,138 patients to evaluate the effect of TxA (pool of CRASH-2 and WOMAN trial subjects). ${ }^{23}$ It found a survival benefit of 70\% in patients who were treated early with TxA. The survival benefit degraded by $10 \%$ for every 15 -minute delay up to 3 hours after which there was no mortality benefit with TxA administration.

TxA is included in the list of essential medicines by the World Health Organization for the management of acute bleeding in patients with trauma, cardiopulmonary bypass, and postpartum hemorrhage, and early administration of TxA is now a part of trauma guidelines worldwide. ${ }^{24}$

\section{Comments}

In the light of the available evidence, it may be prudent to administer TxA $1 \mathrm{~g}$ early ( $\leq 3$ hours of injury) in major trauma to attain maximal benefit.

\section{Traumatic Brain Injury}

\section{Rationale}

Following traumatic brain injury (TBI), patients experience a hypercoagulable state in the early period, followed by an increased fibrinolytic activity. Both hyper- and hypocoagulable states can lead to secondary brain insults. The pathogenesis at the heart of TBI-induced coagulopathy is a matter of extensive debate. Massive release of tissue factor, DIC, hyperfibrinolysis, protein C-thrombomodulin activation, and platelet dysfunction have all been implicated. ${ }^{25}$ TBI-associated coagulopathy results in higher odds of mortality and unfavorable outcome. ${ }^{26}$ Another phenomenon that is unique to TBI is the progression of hemorrhagic lesion by either expansion or development of new, noncontiguous hemorrhages, commonly occurring in the initial 6 to 12 hours of injury and referred to as hemorrhagic progression of contusion (HPC). ${ }^{27}$ In a prospective study of 153 TBI patients, the frequency of early HPC (within 6 hours of primary insult) was $43.5 \%$ and carried a fivefold hazard ratio of unfavorable outcome. ${ }^{28}$ Understanding the pathogenesis of TBI-induced coagulopathy resulted in increased use of procoagulants that include fresh frozen plasma (FFP), cryoprecipitate, platelet concentrate, factor concentrates such as prothrombin complex concentrate (PCC), and pharmaceutical agents such as recombinant factor VII and AFs.

\section{Evidence}

The potential role of TxA in TBI has been explored in two trials till date. IBS was a randomized placebo-controlled trial nested within the CRASH-2 trial that enrolled $270 \mathrm{TBI}$ patients. ${ }^{6}$ The primary outcome studied was total hemorrhage growth on computed tomography (CT) of the head obtained at admission and after 24 to 48 hours. The mean total hemorrhage growth was lower in the TxA group as compared with the placebo group (5.9 $\mathrm{mL}$ [standard deviation (SD) 26.8] vs. $8.1 \mathrm{~mL}$ [SD 29.2]) with the adjusted difference of $-3.8 \mathrm{~mL}$ (95\% CI: -11.5 to 3.9). The other randomized placebo-controlled trial recruited 240 moderate to severe TBI patients (postresuscitation Glasgow coma scale [GCS] 4-12) where a CT scan was obtained within 8 hours of injury. ${ }^{29}$ All patients received $1 \mathrm{~g}$ bolus TxA, followed by $1 \mathrm{~g}$ over 8 hours. Progressive ICH (new lesion or significant growth $\geq 25 \%$ ) was noted in $18 \%$ patients who were treated with TxA as compared with $27 \%$ in the placebo group ( $R R=0.65$; $95 \%$ CI: 0.4-1.05). A Cochrane review pooled the above trials and concluded that in TBI patients, TxA may reduce mortality, but the quality of evidence is low and there is substantial uncertainty. ${ }^{22}$ The clinical implication of these findings is limited as the reduction in mortality may be related to the effect of TxA on associated extracranial injuries.

\section{Ongoing Research}

Currently three trials are underway to investigate the effect of TxA in isolated TBI patients. CRASH-3 trial (NCT01402882) plans to recruit 13,000 isolated TBI patients within 8 hours of injury, and the results are expected by mid-2019. ${ }^{30} \mathrm{~A}$ nested randomized controlled trial (RCT) within the CRASH-3 trial (CRASH-3 IBMS) is the mechanistic substudy to evaluate the effect of TxA on intracranial hemorrhage and cerebral ischemia in isolated TBI. ${ }^{31}$ The other trial is investigating the application of TxA in a prehospital setting in TBI patients within 2 hours of injury (NCT02645552, NCT01990768). ${ }^{32}$

\section{Comments}

The evidence is robust for the use of TxA in TBI patients with associated exsanguinating extracranial injuries where early TxA administration reduces the risk of death. Results from CRASH-3 are expected to throw light on the management of isolated TBI patients. One should keep in mind the possible thrombotic complications in TBI patients when using TxA.

\section{Nontraumatic Intracerebral Hemorrhage}

\section{Rationale}

A recognized modifiable risk factor in $\mathrm{ICH}$ is hematoma expansion (HE), defined as an absolute increase in size by $>12.5 \mathrm{~mL}$ or proportional increase by $\geq 33 \%$, and has been the target of therapeutic interventions..$^{33}$ Approximately onethird of ICH patients experience HE within 6 hours of symptom onset, and it strongly predicts poor outcome. ${ }^{34}$ Strategies to limit HE have primarily focused on lowering blood pressure, whereas a wide variety of hemostatic therapies including TxA have been tried with variable results.

\section{Evidence}

Observational studies have shown less HE with TxA, with $2 \mathrm{~g}$ showing better results than the conventional $1 \mathrm{~g}$ dose. ${ }^{35} \mathrm{~A}$ Cochrane review concluded that TxA led to a nonsignificant 
reduction in $\mathrm{HE}(\mathrm{RR}=0.76$; 95\% $\mathrm{CI}: 0.56-1.05)$ and a nonsignificant increase in death/unfavorable outcome with a relative risk of 1.25 (95\% CI: 0.57-2.75). ${ }^{36}$ Recently published TICH-2 trial, the largest reported trial evaluating TxA in spontaneous ICH, recruited 2,325 patients across 124 hospitals in 12 countries. ${ }^{37}$ The treatment arm received TxA $1 \mathrm{~g}$ over 10 minutes, followed by $1 \mathrm{~g}$ over 8 hours with a matching placebo. The functional status quantified by modified Rankin scale (mRS) and mortality at day 90 did not differ significantly between the treatment and placebo arms (adjusted odds ratio [aOR] for functional status: 0.88 ; 95\% CI: 0.76-1.03; $p=0.11$, aOR for mortality: $0.88,95 \% \mathrm{CI}$ : $0.76-1.03, p=0.11$ ). Notably, adverse events were lower in the treatment arm, and fewer patients in the treatment arm had $\mathrm{HE}$ on day 2 ( $25 \%$ vs. $29 \%$, respectively; aOR $=0.80 ; 95 \%$ CI: $0.66-0.98 ; p=0.03)$. Though this study failed to show improved clinical outcome with TXA in acute ICH patients, it can be speculated that the small perceived benefit may be better reciprocated in patients with larger baseline hematomas, younger population, spot sign positive patients, and earlier administration of TXA (<3-hour window). ${ }^{38}$

\section{Ongoing Research}

Seven RCTs are currently investigating the role of TxA in reducing $\mathrm{ICH}$. These include Stopping Hemorrhage With Tranexamic Acid for Hyperacute Presentation Including Mobile Stroke Units (STOP-MSU NCT03385928), the Spot sign and Tranexamic acid On Preventing ICH growth-Australasia Trial (STOP-AUST NCT01702636), The Norwegian Intracerebral Hemorrhage Trial (NOR-ICH, EudraCT number 2012-005594-30), Tranexamic Acid for Acute ICH Growth prEdicted by Spot Sign (TRAIGE, NCT02625948), Tranexamic acid for IntraCerebral Hemorrhage secondary to Novel Oral AntiCoagulants (TICH-NOAC NCT02866838), Tranexamic Acid for Spontaneous Acute Cerebral Hemorrhage (TRANSACT NCT 03044184), and Tranexamic acid and biomarkers in Emergency management of Spontaneous IntraCerebral Hemorrhage (EsICH). STOP-AUST trial was completed in July 2018, and results were unavailable at the time of writing this review. $^{39}$

\section{Comments}

Though the TICH-2 trial failed to show functional improvement with TxA following ICH, the study showed reduced HE and no increase in adverse events. Until the ongoing research results are published, it is reasonable to consider early TxA administration only in moderate to large acute nontraumatic ICH.

\section{Chronic Subdural Hematoma}

\section{Rationale}

Another area that has invited research on AFs is the CSDH. The pathophysiology of CSDH is complex with an intertwined cascade of inflammation, growth factors upregulation, angiogenesis, recurrent micro bleeds, local coagulopathy, hyperfibrinolysis, and exudation. At the site of CSDH, evidence shows that there is existence of local hyperfibrinolysis with abundance of tPA from adjoining endothelium, low plasminogen levels, and increased fibrinogen degradation products (FDPs) as fibrin monomers and D-dimers when compared with serum. ${ }^{40,41}$ In the light of this evidence, it is hypothesized that TxA might inhibit the fibrinolytic activity and kinin-kallikrein inflammatory cascade-induced vascular permeability, translating into gradual resorption of the subdural hematoma.

\section{Evidence}

In a nonrandomized study on 21 patients with CSDH managed conservatively, TxA was administered orally at a dose of $750 \mathrm{mg} /$ day orally. ${ }^{42}$ The median hematoma volume at presentation was $55.6 \mathrm{~mL}$ (interquartile range [IQR]: 7.5-140.5 $\mathrm{mL})$. At 8 weeks, the median volume for all 21 patients was $3.7 \mathrm{~mL}$ (IQR: 0-22.1 mL). Similar results have been reported in CSDH patients treated with subdural evacuating port system (SEPS), followed by oral TxA postoperatively ${ }^{43}$ Moreover, few case series have reported TxA as an adjunct to surgery, particularly to avoid recurrences and in patients who are at high risk for surgical intervention. ${ }^{44}$

\section{Ongoing Research}

Currently many prospective studies are ongoing in CSDH patients to study the effect of TxA on CSDH resolution, recurrence rates, associated thromboembolic complications, and its benefits on anticoagulated patients. These studies include Tranexamic Acid in Chronic Subdural Hematomas (TRACS) trial (NCT02568124), Tranexamic acid in the Treatment of Residual Chronic Subdural Hematoma (TRACE) trial (NCT03280212), and Tranexamic acid to prevent Operation in Chronic subdural Hematoma (TORCH) trial (NTR 6758), a Dutch study. ${ }^{45}$

\section{Comments}

With the available results that are primarily retrospective, small sample size and with the exclusion of anticoagulated patients, the role of TxA in this setting remains uncertain. Its effect on hematoma resolution/recurrence and adverse thromboembolic events will be clarified with the publication of results of ongoing studies.

\section{Spontaneous Subarachnoid Hemorrhage}

\section{Rationale}

Rerupture of an intracranial aneurysm significantly increases the odds of in-hospital mortality by fivefold and reduces the chance of survival with functional dependence by half. ${ }^{46}$ Rerupture within the first 24 hours following aneurysmal SAH occurs in approximately 9 to $17 \%$ of patients, over twothirds occurring within 3 to 6 hours of index bleed. ${ }^{47}$ Rebleeding is one of the preventable causes of mortality. The use of AFs after SAH was first proposed by Mullan and Dawley in 1968. ${ }^{3}$ Fibrinolytic activation around the clot, formed at the time of initial hemorrhage, was thought to be a culprit in the pathogenesis of rebleed, and AFs were found to be a strong case to mitigate this sinister event. Evidence of reduced CSF plasminogen and elevated FDP levels following 2 weeks of 
TxA treatment in ruptured aneurysm patients further supported this speculation. ${ }^{48}$ Late surgery in aneurysmal SAH being the norm in those times, AFs found a strong foothold in the prevention of rebleed in these patients.

\section{Evidence}

A couple of trials reported a statistically significant reduction in the incidence of rebleeding and a nonsignificant trend toward development of delayed cerebral ischemia. ${ }^{49,50}$ The International Cooperative Study on the timing of aneurysm surgery trial analyzed the outcome data of patients in the early surgery group between those who received AFs versus controls and noted that the cumulative 2-week rebleed rate was lower in the AF group (11.7\%) compared with those who were not administered AFs (19.4\%). However, there was a marked increase in the risk of delayed cerebral ischemia ( $32 \%$ vs. $23 \%$ ), and no differences in the functional outcome were observed between the two groups. ${ }^{4}$ Due to increased incidence of DCI and hydrocephalus, enthusiasm over use of TxA in aneurysmal bleed showed a decadence. ${ }^{51}$ The final nail in the coffin was driven by the Cochrane 2003 review that included 1,399 patients across nine RCTs and noted that AF treatment had no beneficial effect (odds of a poor outcome; $\mathrm{OR}=1.12 ; 95 \% \mathrm{CI}: 0.88-1.43)$. Though there was reduced rebleeding risk (OR $=0.55$; 95\% CI: $0.42-0.71)$ with no significant association with hydrocephalus, the accrued benefit got offset by the increased risk of cerebral ischemia $(O R=1.39$; 95\% CI: 1.07-1.89). ${ }^{52}$ This review soon faced considerable criticism for including decade-old trials when cerebral ischemia preventive therapies were still not in vogue, AF treatment was used for prolonged periods (2-6 weeks) and was initiated well beyond the 72-hour window. Also, the review failed to include a Swedish trial in which TxA was given early to 505 patients and for a short period $(<72$ hours) until aneurysm was secured. ${ }^{53}$ The rate of rebleeding in the treated group was significantly lower when compared with no treatment group ( $2.4 \%$ vs. $10.8 \%, p=0.01)$. Notably, there was no increase in ischemic events or vasospasm noted in the TxA group.

A subsequent Cochrane review in 2013 included 10 trials with 1,904 participants and found no improvement in all-cause mortality or poor outcome with the use of AFs. ${ }^{54}$ Though the reviewers noted a reduced risk of rebleed $(\mathrm{RR}=$ $0.65,95 \%$ CI: 0.44-0.97), they concluded that evidence does not support AFs use in SAH to prevent rebleeding even with the application of cerebral ischemia preventive measures. This Cochrane review also had several limitations such as inclusion of studies in which late surgery was the norm, use of AF for prolonged duration, and lack of consistency on cerebral ischemia diagnosis methodology between studies.

In the current era, early obliteration of aneurysm is recommended to aggressively manage DCI and prevent rebleed. Hence, there is a resurgence of interest in the AF use in the pre-intervention period of ruptured cerebral aneurysms.

A meta-analysis that included 17 studies with 2,872 participants addressed the issue by reviewing these studies in three groups: studies with short-term AF and medical prevention of vasospasm, studies with long-term AF and medical prevention of vasospasm, and studies with long-term AF and no medical prevention of vasospasm. ${ }^{55}$ The reviewers noted that patients receiving short-term AF therapy with cerebral protection strategies had a better outcome with a pooled OR of 0.79 (95\% CI: $0.57-1.08$ ). It may be plausible that shortterm AF ( $<72$ hours) therapy with early aneurysm exclusion, use of aggressive hypertensive therapy, and calcium channel blocker therapy may reduce rebleeding and offset the incidence of delayed cerebral ischemia with improved clinical outcomes. Moreover, the stigma of thrombotic events associated with AF use in spontaneous intracranial bleeding has been cleared, albeit sparingly, by another meta-analysis of 57 studies including 5,049 participants, of which $72 \%$ of patients had a spontaneous SAH. ${ }^{56}$ The incidence of limb ischemia, pulmonary embolism, and myocardial infarction was noted to be around $1 \%$ with TxA use. The rate of DCI, however, was 9.7\% (95\% CI: 5.5-14.8) in SAH patients.

\section{Guidelines}

Antifibrinolytic use is still a gray area in SAH patients. European Stroke Organization guidelines advise against its use and the American Heart Association/American Stroke Association guidelines advocate optional use of short-term ( $<72$ hours) AF therapy with TxA or aminocaproic acid when there is unavoidable delay in aneurysm occlusion and when there are no medical contraindications to AF use. ${ }^{57,58}$ Neurocritical Care Society guidelines also suggest use of AF therapy for less than 72 hours. $^{59}$

\section{Ongoing Research}

An ongoing Dutch trial, ULTRA, endeavors to address the issue of whether ultra early and short-term TxA use added to the standard SAH management will improve functional outcome at 6 months. The study will recruit 950 adult aneurysmal SAH patients randomized to TxA treatment $(1 \mathrm{~g}$ IV immediately followed by $1 \mathrm{~g}$ every 8 hours until aneurysm obliteration or a maximum of 24 hours, whichever is earlier). ${ }^{60}$

\section{Comments}

The available evidence is still sparse and is of low quality. It is clear that rebleeding has a high case fatality rate and morbidity. AFs reduce the rebleeding risk by 35 to $40 \%$. There is no uniform international consensus on TxA use in SAH. Though more conclusive evidence toward an improved clinical outcome is eagerly awaited, in the absence of contraindications to the use of TxA, it is reasonable to consider early and shortterm therapy ( $<72$ hours) if unavoidable delay in securing the aneurysm is anticipated.

\section{Perioperative Period}

The role of TxA in reducing surgical bleed and transfusion requirements has been proven in a variety of perioperative settings. A systematic review of 129 randomized clinical trials including 10,488 patients showed that TxA administration reduced the probability of blood transfusion (pooled risk ratio $\mathrm{RR}=0.62 ; 95 \% \mathrm{CI}: 0.58-0.65 ; p<0.001) .{ }^{61}$ General recommendations for perioperative use include a intravenous 
bolus dose of 10 to $20 \mathrm{mg} / \mathrm{kg}$ prior to skin incision, followed by a maintenance infusion $(1-10 \mathrm{mg} / \mathrm{kg} / \mathrm{h}){ }^{1}$

\section{Intracranial Tumors Rationale}

With the background knowledge of hemostatic dysfunction associated with primary brain tumors, particularly meningiomas, which are known to induce local tPA leading to fibrinolysis, it seems plausible to consider TxA perioperatively. ${ }^{62} \mathrm{TxA}$ administration may provide additional benefit of reducing the incidence of postoperative hematoma, especially in surgical excision of arteriovenous malformations (AVMs), tumors, $\mathrm{TBI}$, and CSDH. Trials on AF use in intracranial tumors were published more than a decade ago. ${ }^{63}$ These, however, failed to generate interest, and publications are restricted to few case reports. ${ }^{64}$ One of the cited reasons for limited scope of prophylactic use of TxA is that not all neurosurgical procedures bleed excessively; the other concern being TxA-associated seizures.

\section{Evidence}

A prospective RCT in 100 adult patients undergoing excision of intracranial tumors noted a statistically significant reduction in total blood loss in TxA group compared with placebo $(817 \mathrm{~mL} \pm 423.3 \mathrm{~mL}$ vs. $1,084 \mathrm{~mL} \pm 604.8 \mathrm{~mL} ; p=0.01) .65$ Though the amount of allogeneic blood transfused was similar in the two groups, hemoglobin decline was significant in the placebo group $(p=0.02)$. The fibrinogen levels postoperatively were significantly elevated in the TxA group supporting the fibrinolysis inhibition theory for reduced blood loss with AFs. Another prospective double-blind RCT randomized 60 patients undergoing excision of large meningiomas (size $>4 \mathrm{~cm}$ ) noted reduced blood loss in TxA group compared with placebo ( $830 \mathrm{~mL}$ vs. $1,124 \mathrm{~mL} ; p=0.03) .{ }^{66} \mathrm{The}$ investigators additionally reported better hemostasis in the TxA group on a 5-grade surgical hemostasis scale $(p=0.007)$. There were no thromboembolic events noted with the use of TxA. Complex skull base surgeries are another unique group with significant blood loss due to problematic surgical access and extension of tumor pathology into neighboring sinuses. A retrospective study of 245 patients with skull base tumors who received TXA was compared with matching control (274 patients). ${ }^{67}$ TxA-treated patients had a 6\% reduction in the absolute risk of transfusion.

Application of TxA topically at surgical site, though prevalent in other surgical scenarios, in intracranial surgery, has been limited because of its tendency to induce seizures.

\section{Ongoing Research}

A phase 3 clinical trial on efficacy of TxA in brain tumor resection (NCT01655927) intends to determine whether TxA is effective or not in the reduction of intraoperative blood loss in brain tumor resections.

\section{Procedures on Spine \\ Rationale}

Spine surgery is unique considering that it entails stripping the paraspinal muscles off the lamina leaving raw muscle edges and periosteum to ooze. The older adults with thin periosteum and osteoporotic bone with large vascular channels tend to bleed more. Additionally, adult spine surgery usually involves instrumentation at several segments and the rate of revision surgery is high. Whereas laminectomy is a low transfusion risk procedure, spinal fusion surgery entails a blood loss approximating $800 \mathrm{~mL}$ in noninstrumental fusion versus over $1.5 \mathrm{~L}$ in instrumental fusions.$^{68}$ Overall, perioperative blood loss and patient outcome in spine surgery are related to the duration of surgery, anterior-posterior sequential approach, number of vertebrae fused, number of osteotomies, and protocol-based transfusion practice. Moreover, the ensuing coagulopathy can lead to postoperative neuraxial hematoma with potential neurologic compromise. ${ }^{69}$

\section{Evidence}

A study in spine patients included 147 adult patients undergoing elective posterior thoracic/lumbar instrumented spinal fusions and noted one-third reduction in perioperative blood loss, but its efficacy on allogeneic transfusion requirement and clinical outcome failed to reach significance. ${ }^{70}$ Subsequent trials showed contradictory findings for outcomes of blood loss and blood transfusion. ${ }^{71,72}$ A 2017 meta-analysis comprehensively explored the efficacy and safety of AFs in spine surgery. ${ }^{73}$ It included 11 studies and showed reduced total blood loss (standard mean difference [SMD] $-0.75 ; 95 \% \mathrm{CI}:-0.93$ to $-0.57 ; p=0.000$ ). The intraoperative and postoperative blood losses were significantly reduced with TxA. However, all studies had significant heterogeneity for the studied outcomes regarding drug dosage regimes, surgical procedure, duration of surgery, methods of blood loss estimation, transfusion trigger, and transfusion protocols. A recent multicenter, prospective randomized double-blind trial compared TxA with placebo in posterior instrumentation spine surgery $(>3$ segmental fusions) for efficacy based on number of transfused units and perioperative blood loss. ${ }^{74}$ There was significant reduction in the need for transfusion ( $42 \%$ in TxA vs. $67 \%$ in placebo group; $p<0.05$ ) and intraoperative blood loss.

With the current evidence, the European Society of Anaesthesiology recommends TxA administration to attenuate perioperative blood loss in orthopedic and spine surgery if the risk-benefit ratio is favorable. ${ }^{75}$ Caution is advocated in women, previous history of venous thromboembolism, and older adults (age above 60 years).

Another aspect of TxA use that is gaining popularity is its topical application into the surgical wound site. Intuitively, the lower plasma TxA levels and thromboembolic complications are advantageous. ${ }^{76}$ Though there is literature supporting this topical application in varied surgical settings, the literature in spine procedures are limited. ${ }^{77}$

Currently, there is interest in combination therapy with topical TxA added to systemic TxA.

\section{Ongoing Research}

There are currently two ongoing trials to delineate the optimum dose of TxA required to achieve maximal benefit with minimal harm ClinicalTrials.gov NCT 02053363 and NCT02188576. ${ }^{78}$ 


\section{Comments}

TxA can be administered in major complex multilevel spine surgery, revision surgery, prolonged procedures, and where substantial blood loss is expected (> 20\% total blood volume), provided no contraindications exist. The optimal dose for surgery remains unclear; however, a loading dose of $1 \mathrm{~g}$ may be reasonable followed by maintenance infusion. Topical TxA may be a reasonable choice to consider in the wake of proven safety and efficacy profile in cardiac surgical settings.

\section{Safety Concerns}

The adverse events of TxA are seen with prolonged oral administration and include headache, nausea, vomiting, diarrhea, dyspepsia, dysmenorrhea, dizziness, back pain, numbness, phosphenes, and anemia. ${ }^{79} \mathrm{TxA}$ is contraindicated if there is a history of preexisting active thromboembolic event/disorder, DIC, renal failure, and recent coronary or vascular stent. ${ }^{80}$ The two common worrisome adverse events are thrombotic risks and seizures.

\section{Thrombotic Complications}

TxA-induced fibrinolytic inhibition theoretically entails a prothrombotic state and a plausible concern for thrombotic complications such as deep vein thrombosis (DVT), pulmonary embolism (PE), myocardial infarction (MI), renal cortical necrosis, and stroke. Though the majority of these are reported as case reports and case series, large trials have refuted this concern. ${ }^{81}$ With the available evidence in trauma and perioperative scenario, the thrombotic potential of TxA remains more of a theoretical concern.

The neurosurgical patients are unique in this aspect as the reported incidence of DVT is between 18 and 50\% and PE in 0 to $25 \%$ patients. ${ }^{82}$ Additionally, there is risk of delayed cerebral ischemia seen with wide range of neuropathologies such as SAH, and TBI.

In the meta-analyses of patients undergoing arthroplasties and prostate cancer patients, who are inherently at a high risk of thromboembolic events, no increased incidence of VTE was noted. ${ }^{83}$ Even following a high-dose administration of TxA (50-100 mg/kg), 30-day thrombotic events were similar to the placebo group. ${ }^{84}$

\section{Seizures}

Epileptogenic potential of cortical TxA has been documented in animal studies and with accidental intrathecal TxA administration. ${ }^{85}$ Typically, TxA-associated seizures are myoclonic or generalized tonic-clonic seizures occurring within the first 5 to 8 hours of surgery as the patient is weaned off the anesthetics. ${ }^{86}$ A plausible mechanism is elucidated by the remarkable structural homology between TxA, gamma-aminobutyric acid type $A\left(G_{A B A}\right)$, and glycine receptors. It is possible that TxA acts as a competitive antagonist of GABA type $A$ receptors and blocks the chloride channels responsible for neuronal hyperpolarization.

This manifests as increased excitability of the neuronal networks resulting myoclonus. ${ }^{87,88}$ Finally, owing to the high affinity of TxA for glycine receptors, it is suggested that the proconvulsant effects are probably mediated by the disinhibition of the glycine receptor-mediated tonic current in the neuronal circuitry. ${ }^{89}$ The incidence rate of TxA-associated seizures was noted to be dose dependent with incidence rate varying from $1.4 \%$ in low-dose group to $5.3 \%$ in the highdose group..$^{90}$ High incidence rate in cardiac surgical patients might be due to the frequent coexistence of renal dysfunction in these patients. ${ }^{91,92}$ The relationship between TxA and seizure activity in noncardiac surgery populations remains undefined. An odd case report of postoperative seizure in a neurosurgical patient who received TxA intraoperatively was attributed to TxA. ${ }^{93}$

However, the occurrence of seizures has not been noted in the CRASH-2 trial.

\section{Future Research}

The research into optimal use of AFS is a steep uphill task and needs to define the optimal dosage of TxA and possible mechanisms of mortality benefit with its use. Dose regimens are varied, and there are no in vivo pharmacokinetic studies to support any of the regimens. Low doses tend to be questionable in terms of reducing allogeneic transfusion but may tend to cause less harm compared with high doses. Another related issue is to find out the minimum therapeutic concentration to bring about clinical inhibition of fibrinolysis and titrate the dosage regimens accordingly.

Second, there is some evidence suggesting increased mortality with inadvertent use of TxA in patients with bleeding trauma. In a database of 2,540 trauma patients, different fibrinolytic phenotypes were noted, fibrinolysis shutdown being most common (46\%), followed by physiologic fibrinolysis (36\%) and hyperfibrinolysis (18\%). ${ }^{94}$ In patients who received TxA, mortality increased in normal fibrinolysis and shutdown subtypes, and there was no mortality benefit in hyperfibrinolysis phenotypes.95,96 An evidence-based approach to the use of TxA based on hyperfibrinolysis assessment is the need of the hour.

A randomized placebo-controlled TAMPITI (Tranexamic Acid Mechanisms and Pharmacokinetics in Traumatic Injury) trial (NCT02535949) intends to evaluate the effects of TxA on the immune system (viz. activated monocytes, cytokines), pharmacokinetics, safety and efficacy of two different doses of TxA in severely injured trauma patients. ${ }^{97}$

Third, evidence is robust denying a prothrombotic potential of TxA in trauma and other perioperative settings. However, there are sparse data on specific patient population who are at higher risk for thromboembolic events, which may be the case in neurocritical care settings. This has been primarily because expert consensus and drug formulary contraindicate its use in this subset of patients.

Fourth, in the absence of any direct measurements of TxA effect, surrogate measures such as reduced allogeneic transfusion have shown wide variability in patients and different surgical settings. It is likely that genetic variability may be responsible. ${ }^{98}$

Finally, in the absence of ready availability of direct tests of fibrinolysis and poor reliability of standard laboratory tests in detecting fibrinolysis, this remains a major gray 
area for ubiquitous administration of TxA. Though rotational ROTEM/thromboelastography (TEG) is the most widely used clinical test to detect hyperfibrinolysis, the sensitivity is poor in detecting mild to moderate degrees of fibrinolytic activation. ${ }^{19}$

An interesting speculation that is gaining momentum is the anti-inflammatory and antineoplastic potential of TxA by inhibiting plasminogen and plasmin at high doses. ${ }^{99}$

\section{Conclusion}

Tranexamic acid as a fibrinolytic inhibitor appears promising in exsanguinating trauma, postpartum hemorrhage, and cardiopulmonary bypass-dependent cardiac surgery with a reasonable safety profile. However, the evidence for universal application in neuroanesthesia and neurocritical care is less robust. As the results of various ongoing trials are awaited, there are few, if any, level I recommendations for TxA use in neurosurgical patients. It may have a case where the benefits justify the harm as in large vascular intracranial tumors, multilevel spine corrective surgery, pre-intervention period in aSAH, and TBI with significant extracranial exsanguinating trauma. Basic premise that needs to be followed is "give early, stay low." The picture is elusive until the results of CRASH-3 trial throw some light on the future of TxA in neuroanesthesia and neurocritical care.

\section{Conflict of Interest}

None declared.

\section{References}

1 Dunn CJ, Goa KL. Tranexamic acid: a review of its use in surgery and other indications. Drugs 1999;57(6):1005-1032

2 Schloss B, Gulati P, Yu L, et al. Impact of aprotinin and renal function on mortality: a retrospective single center analysis. J Cardiothorac Surg 2011;6:103

3 Mullan S, Dawley J. Antifibrinolytic therapy for intracranial aneurysms. J Neurosurg 1968;28(1):21-23

4 Kassell NF, Torner JC, Jane JA. Haley EC Jr, Adams HP. The international cooperative study on the timing of aneurysm surgery. Part 2. Surgical results. J Neurosurg 1990;73(1):37-47

5 Shakur H, Roberts I, Bautista R, et al; CRASH-2 trial collaborators. Effects of tranexamic acid on death, vascular occlusive events, and blood transfusion in trauma patients with significant haemorrhage (CRASH-2): a randomised, placebocontrolled trial. Lancet 2010;376(9734):23-32

6 CRASH-2 Collaborators, Intracranial Bleeding Study. Effect of tranexamic acid in traumatic brain injury: a nested randomised, placebo controlled trial (CRASH-2 Intracranial Bleeding Study) BMJ 2011;343:d3795

7 Chapin JC, Hajjar KA. Fibrinolysis and the control of blood coagulation. Blood Rev 2015;29(1):17-24

8 Henry DA, Carless PA, Moxey AJ, et al. Anti-fibrinolytic use for minimising perioperative allogeneic blood transfusion. Cochrane Database Syst Rev 2011;CD001886

9 OkamotoS, Sato S, Takada Y, Okamoto U. An active stereo-isomer (trans-form) of AMCHA and its antifibrinolytic (antiplasminic) action in vitro and in vivo. Keio J Med 1964;13:177-185

10 Melander B, Gliniecki G, Granstrand B, Hanshoff G. Biochemistry and toxicology of amikapron; the antifibrinolytically active isomer of AMCHA. (A comparative study with. $\varepsilon$-aminocaproic acid) Acta Pharmacol Toxicol (Copenh) 1965;22(4):340-352
11 Nilsson IM. Clinical pharmacology of aminocaproic and tranexamic acids. J Clin Pathol Suppl ( $\mathrm{R}$ Coll Pathol) 1980;14:41-47

12 Andersson L, Nilsson IM, Niléhn JE, Hedner U, Granstrand B, Melander B. Experimental and clinical studies on AMCA, the antifibrinolytically active isomer of p-aminomethyl cyclohexane carboxylic acid. Scand J Haematol 1965;2(3):230-247

13 Horrow JC, Van Riper DF, Strong MD, Grunewald KE, Parmet JL. The dose-response relationship of tranexamic acid. Anesthesiology 1995;82(2):383-392

14 Ker K, Prieto-Merino D, Roberts I. Systematic review, meta-analysis and meta-regression of the effect of tranexamic acid on surgical blood loss. Br J Surg 2013;100(10):1271-1279

15 Grassin-Delyle S, Theusinger OM, Albrecht R, et al. Optimisation of the dosage of tranexamic acid in trauma patients with population pharmacokinetic analysis. Anaesthesia 2018;73(6):719-729

16 Nishida T, Kinoshita T, Yamakawa K. Tranexamic acid and trauma-induced coagulopathy. J Intensive Care 2017;5:5

17 Brohi K, Singh J, Heron M, Coats T. Acute traumatic coagulopathy. J Trauma 2003;54(6):1127-1130

18 Kauvar DS, Lefering R, Wade CE. Impact of hemorrhage on trauma outcome: an overview of epidemiology, clinical presentations, and therapeutic considerations. J Trauma 2006;60(6, Suppl):S3-S11

19 Raza I, Davenport R, Rourke C, et al. The incidence and magnitude of fibrinolytic activation in trauma patients. J Thromb Haemost 2013;11(2):307-314

20 Roberts I, Shakur H, Afolabi A, et al; CRASH-2 collaborators. The importance of early treatment with tranexamic acid in bleeding trauma patients: an exploratory analysis of the CRASH-2 randomised controlled trial. Lancet 2011;377(9771): 1096-1101. e2

21 Morrison JJ, Dubose JJ, Rasmussen TE, Midwinter MJ. Military Application of Tranexamic Acid in Trauma Emergency Resuscitation (MATTERs) study. Arch Surg 2012;147(2):113-119

22 Ker K, Roberts I, Shakur H, Coats TJ. Antifibrinolytic drugs for acute traumatic injury. Cochrane Database Syst Rev 2015;5(5):CD004896

23 Gayet-Ageron A, Prieto-Merino D, Ker K, Shakur H, Ageron FX, Roberts I; Antifibrinolytic Trials Collaboration. Effect of treatment delay on the effectiveness and safety of antifibrinolytics in acute severe haemorrhage: a meta-analysis of individual patient-level data from 40138 bleeding patients. Lancet 2018;391(10116):125-132

24 Roberts I, Kawahara T, Proposal for the Inclusion of Tranexamic Acid (Antifibrinolytic-lysine Analogue) in the WHO Model List of Essential Medicines. Geneva, Switzerland: World Health Organization;2010

25 Nakae R, Takayama Y, Kuwamoto K, Naoe Y, Sato H, Yokota H. Time course of coagulation and fibrinolytic parameters in patients with traumatic brain injury. J Neurotrauma 2016;33(7):688-695

26 Epstein DS, Mitra B, O'Reilly G, Rosenfeld JV, Cameron PA. Acute traumatic coagulopathy in the setting of isolated traumatic brain injury: a systematic review and meta-analysis. Injury 2014;45(5):819-824

27 Kurland D, Hong C, Aarabi B, Gerzanich V, Simard JM. Hemorrhagic progression of a contusion after traumatic brain injury: a review. J Neurotrauma 2012;29(1):19-31

28 Juratli TA, Zang B, Litz RJ, et al. Early hemorrhagic progression of traumatic brain contusions: frequency, correlation with coagulation disorders, and patient outcome: a prospective study. J Neurotrauma 2014;31(17):1521-1527

29 Yutthakasemsunt S, Kittiwatanagul W, Piyavechvirat P, Thinkamrop B, Phuenpathom N, Lumbiganon P. Tranexamic acid for patients with traumatic brain injury: a randomized, doubleblinded, placebo-controlled trial. BMC Emerg Med 2013;13:20 
30 Dewan Y, Komolafe EO, Mejía-Mantilla JH, Perel P, Roberts I, Shakur H; CRASH-3 Collaborators. CRASH-3-tranexamic acid for the treatment of significant traumatic brain injury: study protocol for an international randomized, double-blind, placebo-controlled trial. Trials 2012;13:87

31 Mahmood A, Roberts I, Shakur H. A nested mechanistic substudy into the effect of tranexamic acid versus placebo on intracranial haemorrhage and cerebral ischaemia in isolated traumatic brain injury: study protocol for a randomised controlled trial (CRASH-3 Trial Intracranial Bleeding Mechanistic Sub-Study [CRASH-3 IBMS]) Trials 2017;18(1):330

32 Napolitano LM. Prehospital tranexamic acid: what is the current evidence? Trauma Surg Acute Care Open 2017;2(1):e000056

33 Davis SM, Broderick J, Hennerici M, et al; Recombinant Activated Factor VII Intracerebral Hemorrhage Trial Investigators. Hematoma growth is a determinant of mortality and poor outcome after intracerebral hemorrhage. Neurology 2006;66(8):1175-1181

34 Kazui S, Naritomi H, Yamamoto H, Sawada T, Yamaguchi T. Enlargement of spontaneous intracerebral hemorrhage. Incidence and time course. Stroke 1996;27(10):1783-1787

35 Law ZK, Meretoja A, Engelter ST, et al. Treatment of intracerebral haemorrhage with tranexamic acid-a review of current evidence and ongoing trials. Eur Stroke J 2017;2(1):13-22

36 Al-Shahi Salman R, Law ZK, Bath PM, Steiner T, Sprigg N. Haemostatic therapies for acute spontaneous intracerebral haemorrhage. Cochrane Database Syst Rev 2018;4:CD005951

37 Sprigg N, Flaherty K, Appleton JP, et al; TICH-2 Investigators. Tranexamic acid for hyperacute primary IntraCerebral Haemorrhage(TICH-2): an international randomised, placebo-controlled, phase 3 superiority trial. Lancet 2018;39110135:2107-2115

38 Broderick JP. Haemostatic treatment for intracerebral haemorrhage. Lancet 2018;39110135:2081-2082

39 Meretoja A, Churilov L, Campbell BCV, et al. The Spot sign and Tranexamic acid On Preventing ICH growth-AUStralasia Trial (STOP-AUST): protocol of a phase II randomized, placebo-controlled, double-blind, multicenter trial. Int J. Stroke 2014;9(4):519-524

40 Ito H, Saito K, Yamamoto S, Hasegawa T. Tissue-type plasminogen activator in the chronic subdural hematoma. Surg Neurol 1988;30(3):175-179

41 Saito K, Ito H, Hasegawa T, Yamamoto S. Plasmin-alpha 2-plasmin inhibitor complex and alpha 2-plasmin inhibitor in chronic subdural hematoma. J Neurosurg 1989;70(1):68-72

42 Kageyama H, Toyooka T, Tsuzuki N, Oka K. Nonsurgical treatment of chronic subdural hematoma with tranexamic acid. J Neurosurg 2013;119(2):332-337

43 Tanweer O, Frisoli FA, Bravate C, et al. Tranexamic acid for treatment of residual subdural hematoma after bedside twistdrill evacuation. World Neurosurg 2016;91:29-33

44 Stary JM, Hutchins L, Vega RA. Tranexamic acid for recurring subdural hematomas following surgical evacuation: a clinical case series. J Neurol Surg A Cent Eur Neurosurg 2016;77(5):422-426

45 Holl DC, Volovici V, Dirven CMF, et al; Dutch Chronic Subdural Hematoma Research Group (DSHR). Pathophysiology and nonsurgical treatment of chronic subdural hematoma: from past to present to future. World Neurosurg 2018;116:402-411.e2

46 Naidech AM, Janjua N, Kreiter KT, et al. Predictors and impact of aneurysm rebleeding after subarachnoid hemorrhage. Arch Neurol 2005;62(3):410-416

47 Germans MR, Coert BA, Vandertop WP, Verbaan D. Time intervals from subarachnoid hemorrhage to rebleed. J Neurol 2014;261(7):1425-1431

48 Fodstad H, Nilsson IM. Coagulation and fibrinolysis in blood and cerebrospinal fluid after aneurysmal subarachnoid haemorrhage: effect of tranexamic acid (AMCA) Acta Neurochir (Wien) 1981;56(1-2):25-38
49 Fodstad H., Tranexamic acid (AMCA) in aneurysmal subarachnoid haemorrhage. J Clin Pathol Suppl (Roy Coll Path)198014: (22 Suppl):68-73

50 Maurice-Williams RS. Prolonged antifibrinolysis: an effective non-surgical treatment for ruptured intracranial aneurysms? BMJ 1978;16118:945-947

51 Roos Y; STAR Study Group. Antifibrinolytic treatment in subarachnoid hemorrhage: a randomized placebo-controlled trial. Neurology 2000;54(1):77-82

52 Roos YB, Rinkel GJ, Vermeulen M, Algra A, van Gijn J. Antifibrinolytic therapy for aneurysmal subarachnoid haemorrhage. Cochrane Database Syst Rev 2003; ( 2):CD001245

53 Hillman J, Fridriksson S, Nilsson O, Yu Z, Saveland H, Jakobsson KE. Immediate administration of tranexamic acid and reduced incidence of early rebleeding after aneurysmal subarachnoid hemorrhage: a prospective randomized study. J Neurosurg 2002;97(4):771-778

54 Baharoglu MI, Germans MR, Rinkel GJE, et al. Antifibrinolytic therapy for aneurysmal subarachnoid haemorrhage. Cochrane Database Syst Rev 2013;8(8):CD001245

55 Gaberel T, Magheru C, Emery E, Derlon JM. Antifibrinolytic therapy in the management of aneurismal subarachnoid hemorrhage revisited. A meta-analysis. Acta Neurochir (Wien) 2012;154(1):1-9

56 Ross J, Al-Shahi Salman R. The frequency of thrombotic events among adults given antifibrinolytic drugs for spontaneous bleeding: systematic review and meta-analysis of observational studies and randomized trials. Curr Drug Saf 2012;7(1):44-54

57 Steiner T, Juvela S, Unterberg A, Jung C, Forsting M, Rinkel G; European Stroke Organization. European Stroke Organization guidelines for the management of intracranial aneurysms and subarachnoid haemorrhage. Cerebrovasc Dis 2013;35(2):93-112

58 Connolly ES. Rabinstein AA, Carhuapoma JR, et al. American Heart Association Stroke Council, Council on Cardiovascular Radiology and Intervention; Council on Cardiovascular Nursing; Council on Cardiovascular Surgery and Anesthesia; Council on Clinical Cardiology. Guidelines for the management of aneurysmal subarachnoid hemorrhage: a guideline for healthcare professionals from the American Heart Association/ American Stroke Association. Stroke 2012;43(6):1711-1737

59 Diringer MN, Bleck TP, Claude Hemphill J, III; et al; Neurocritical Care Society, Critical care management of patients following aneurysmal subarachnoid hemorrhage: recommendations from the Neurocritical Care Society's Multidisciplinary Consensus Conference. Neurocrit Care 2011;15(2):211-240

60 Germans MR, Post R, Coert BA, Rinkel GJ, Vandertop WP, Verbaan D. Ultra-early tranexamic acid after subarachnoid hemorrhage (ULTRA): study protocol for a randomized controlled trial. Trials 2013;14:143

61 Ker K, Edwards P, Perel P, Shakur H, Roberts I. Effect of tranexamic acid on surgical bleeding: systematic review and cumulative meta-analysis. BMJ 2012;344:e3054

62 Tsuda H, Oka K, Noutsuka Y, Sueishi K. Tissue-type plasminogen activator in patients with intracranial meningiomas. Thromb Haemost 1988;60(3):508-513

63 Palmer JD, Francis JL, Pickard JD, Iannotti F. The efficacy and safety of aprotinin for hemostasis during intracranial surgery. J Neurosurg 2003;98(6):1208-1216

64 Bharath K, Bhagat H, Mohindra S. Use of tranexamic acid as a rescue measure to achieve hemostasis after massive blood loss in a pediatric neurosurgical patient. J Neurosurg Anesthesiol 2011;23(4):376-377

65 Vel R, Udupi BP, Satya Prakash MVS, Adinarayanan S, Mishra S, Babu L. Effect of low dose tranexamic acid on intra-operative blood loss in neurosurgical patients. Saudi J Anaesth 2015;9(1):42-48 
66 Hooda B, Chouhan RS, Rath GP, Bithal PK, Suri A, Lamsal R. Effect of tranexamic acid on intraoperative blood loss and transfusion requirements in patients undergoing excision of intracranial meningioma. J Clin Neurosci 2017;41:132-138

67 Mebel D, Akagami R, Flexman AM. Use of tranexamic acid is associated with reduced blood product transfusion in complex skull base neurosurgical procedures: a retrospective cohort study. Anesth Analg 2016;122(2):503-508

68 Möller H, Hedlund R. Instrumented and noninstrumented posterolateral fusion in adult spondylolisthesis-a prospective randomized study: part 2. Spine 2000;25(13):1716-1721

69 Sokolowski MJ, Garvey TA, Perl J, II. et al. Prospective study of postoperative lumbar epidural hematoma: incidence and risk factors. Spine 2008;33(1):108-113

70 Wong J, El Beheiry H, Rampersaud YR, et al. Tranexamic acid reduces perioperative blood loss in adult patients having spinal fusion surgery. Anesth Analg 2008;107(5):1479-1486

71 Elwatidy S, Jamjoom Z, Elgamal E, Zakaria A, Turkistani A, El-Dawlatly A. Efficacy and safety of prophylactic large dose of tranexamic acid in spine surgery: a prospective, randomized, double-blind, placebo-controlled study. Spine 2008;33(24):2577-2580

72 Farrokhi MR, Kazemi AP, Eftekharian HR, Akbari K. Efficacy of prophylactic low dose of tranexamic acid in spinal fixation surgery: a randomized clinical trial. J Neurosurg Anesthesiol 2011;23(4):290-296

73 Li G, Sun TW, Luo G, Zhang C. Efficacy of antifibrinolytic agents on surgical bleeding and transfusion requirements in spine surgery: a meta-analysis. Eur Spine J 2017;26(1):140-154

74 Colomina MJ, Koo M, Basora M, Pizones J, Mora L, Bagó J. Intraoperative tranexamic acid use in major spine surgery in adults: a multicentre, randomized, placebo-controlled trial. $\mathrm{Br}$ J Anaesth 2017;118(3):380-390

75 Kozek-Langenecker SA, Afshari A, Albaladejo P, et al. Management of severe perioperative bleeding: guidelines from the European Society of Anaesthesiology. Eur J Anaesthesiol 2013;30(6):270-382

76 Ipema HJ, Tanzi MG. Use of topical tranexamic acid or aminocaproic acid to prevent bleeding after major surgical procedures. Ann Pharmacother 2012;46(1):97-107

77 Winter SF, Santaguida C, Wong J, Fehlings MG. Systemic and topical use of tranexamic acid in spinal surgery: a systematic review. Global Spine J 2016;6(3):284-295

78 Verma K, Kohan E, Ames CP, et al. A comparison of two different dosing protocols for tranexamic acid in posterior spinal fusion for spinal deformity: a prospective, randomized trial. Int J Spine Surg 2015;9:65

79 CYKLOKAPRON tranexamic acid injection. Antifibrinolytic agent. Available at: https://www.accessdata.fda.gov/drugsatfda_docs/label/2011/019281s030lbl.pdf. Accessed June 15, 2019

80 Calapai G, Gangemi S, Mannucci C, et al. Systematic review of tranexamic acid adverse reactions. J Pharmacovigil 2015;3:171

81 Thiagarajamurthy S, Levine A, Dunning J. Does prophylactic tranexamic acid safely reduce bleeding without increasing thrombotic complications in patients undergoing cardiac surgery? Interact Cardiovasc Thorac Surg 2004;3(3):489-494 [Internet]

82 Khaldi A, Helo N, Schneck MJ, Origitano TC. Venous thromboembolis deep venous thrombosis and pulmonary embolism in a neurosurgical population. J Neurosurg 2011;114(1):40-46

83 Montroy J, Fergusson NA, Hutton B, et al. The safety and efficacy of lysine analogues in cancer patients: a systematic review and meta-analysis. Transfus Med Rev 2017;31(3):141-148
84 Myles PS, Smith JA, Forbes A, et al; ATACAS Investigators of the ANZCA Clinical Trials Network. Tranexamic acid in patients undergoing coronary-artery surgery. N Engl J Med 2017;376(2):136-148

85 Pellegrini A, Giaretta D, Chemello R, Zanotto L, Testa G. Feline generalized epilepsy induced by tranexamic acid (AMCA) Epilepsia 1982;23(1):35-45

86 Sharma V, Katznelson R, Jerath A, et al. The association between tranexamic acid and convulsive seizures after cardiac surgery: a multivariate analysis in 11529 patients. Anaesthesia 2014;69(2):124-130

87 Kratzer S, Irl H, Mattusch C, et al. Tranexamic acid impairs. $\gamma$-aminobutyric acid receptor type A-mediated synaptic transmission in the murine amygdala: a potential mechanism for drug-induced seizures? Anesthesiology 2014;120(3):639-649

88 Furtmüller R, Schlag MG, Berger M, et al. Tranexamic acid, a widely used antifibrinolytic agent, causes convulsions by a gamma-aminobutyric acid(A) receptor antagonistic effect. J Pharmacol Exp Ther 2002;301(1):168-173

89 Lecker I, Wang DS, Romaschin AD, Peterson M, Mazer CD, Orser BA. Tranexamic acid concentrations associated with human seizures inhibit glycine receptors. J Clin Invest 2012;122(12):4654-4666

90 Takagi H, Ando T, Umemoto T; All-Literature Investigation of Cardiovascular Evidence (ALICE) group. Seizures associated with tranexamic acid for cardiac surgery: a meta-analysis of randomized and non-randomized studies. J Cardiovasc Surg (Torino) 2017;58(4):633-641

91 Montes FR, Pardo DF, Carreño M, Arciniegas C, Dennis RJ, Umaña JP. Risk factors associated with postoperative seizures in patients undergoing cardiac surgery who received tranexamic acid: a case-control study. Ann Card Anaesth 2012;15(1):6-12

92 Hui AC, Wong TY, Chow KM, Szeto CC. Multifocal myoclonus secondary to tranexamic acid. J Neurol Neurosurg Psychiatry 2003;74(4):547

93 Merriman B, Mayson K, Sawka A, Akagami R, Flexman AM. Postoperative seizure in a neurosurgical patient: should tranexamic acid be on the differential? Can J Anaesth 2013;60(5):506-507

94 Moore HB, Moore EE, Liras IN, et al. Acute fibrinolysis shutdown after injury occurs frequently and increases mortality: a multicenter evaluation of 2,540 severely injured patients. J Am Coll Surg 2016;222(4):347-355

95 Harvin JA, Peirce CA, Mims MM, et al. The impact of tranexamic acid on mortality in injured patients with hyperfibrinolysis. J Trauma Acute Care Surg 2015;78(5): 905-909,discussion909-911

96 Moore HB, Moore EE, Huebner BR, et al. Tranexamic acid is associated with increased mortality in patients with physiological fibrinolysis. J Surg Res 2017;220:438-443

97 Spinella PC, Bochicchio GV, Tranexamic Acid Mechanisms and Pharmacokinetics in Traumatic Injury (TAMPITI trial). Available athttp://www.tampiti.wustl. edu. Accessed April 16, 2016

98 Iribarren JL, Jimenez JJ, Hernández D, et al. Postoperative bleeding in cardiac surgery: the role of tranexamic acid in patients homozygous for the $5 \mathrm{G}$ polymorphism of the plasminogen activator inhibitor-1 gene. Anesthesiology 2008;108(4):596-602

99 Godier A, Hunt BJ. Plasminogen receptors and their role in the pathogenesis of inflammatory, autoimmune and malignant disease. J Thromb Haemost 2013;11(1):26-34 